\title{
Educação em saúde na assistência pré-natal: revisão de literatura
}

\author{
Health education in prenatal assistance: literature review \\ Educación en salud en la asistencia prenatal: revisión de literatura
}

Raquel Ferreira Cardoso ${ }^{11}$, Vitor Hugo Pantoja Souza1, Thayse Reis Paiva ${ }^{1}$, Danielle Etienne de Oliveira Bezerra Lima ${ }^{1}$, Josias Botelho da Costa ${ }^{1}$, Lohanna Rafaelle Lima de Oliveira ${ }^{1}$, Sara Edyele Santos Marques $^{2}$, Paula Danniele dos Santos Dias², Flávia Andrea Costa da Silva², Danielly do Vale Pereira ${ }^{3^{*}}$.

\section{RESUMO}

Objetivo: Apresentar a importância da educação em saúde, como a equipe multiprofissional a utiliza e as dificuldades que emergem dessa prática na assistência pré-natal. Método: Trata-se de uma revisão narrativa de literatura, nesta a busca dos artigos ocorreu por meio da Biblioteca Virtual em Saúde, no período de novembro de 2017 a janeiro de 2018. Resultados e Discussão: No cruzamento dos descritores obteve-se 748 artigos, após aplicação dos critérios de inclusão e exclusão, foram selecionados 09 artigos indexados nas bases de dados LILACS e BDENF, no período de 2006 a 2017. Os resultados evidenciaram que a educação em saúde proporciona inúmeros benefícios, porém, não é uma tarefa fácil, uma vez que não se limita apenas à transmissão de informações às usuárias, mas de um processo educativo dialógico na busca de autonomia. Considerações finais: Para tal, faz-se importante adoção de estratégias e práticas metodológicas ativas, além da conscientização dos profissionais sobre a importância da educação em saúde no processo de humanização da assistência.

Palavras-Chave: Educação em saúde, Assistência pré-natal e Enfermagem.

\begin{abstract}
Objective: The objective was to present the importance of health education, as the multiprofessional team uses it and the difficulties that emerge from this practice in prenatal care. Method: It is a narrative review of literature, in which the search for the articles occurred through the Virtual Health Library, from November 2017 to January 2018. Results and Discussion: At the intersection of the descriptors, 748 articles were obtained, after applying the criteria of inclusion and exclusion, we selected 09 articles indexed in the databases LILACS and BDENF, from 2006 to 2017. The results showed that health education offers numerous benefits, however, it is not an easy task, since it is not limited only to the transmission of information to users, but a dialogical educational process in the search for autonomy. Final considerations: To this end, it is important to adopt active methodological strategies and practices, as well as the awareness of professionals about the importance of health education in the process of humanization of care.
\end{abstract}

Key words: Health education, Prenatal care and Nursing.

\section{RESUMEN}

Objetivo: Presentar la importancia de la educación en salud, como el equipo multiprofesional la utiliza y las dificultades que emergen de esa práctica en la asistencia prenatal. Método: Se trata de una revisión narrativa de literatura, en esta búsqueda de los artículos ocurrió por medio de la Biblioteca Virtual en Salud, en el

1 Universidade do Estado do Pará (UEPA), Belém-PA.

${ }^{2}$ Universidade Federal do Pará (UFPA), Belém-PA.

${ }^{3}$ Faculdade Metropolitana da Amazônia (FAMAZ), Belém-PA. *E-mail: daniellydovale@hotmail.com

SUBMETIDO EM: 1/2019

ACEITO EM: 2/2019

PUBLICADO EM: 5/2019

REAS/EJCH | Vol. Sup. 23 | e397 | DOI: https://doi.org/10.25248/reas.e397.2019 Página 1 de 8 
período de noviembre de 2017 a enero de 2018. Resultados y Discusión: En el cruce de los descriptores se obtuvo 748 artículos, después de la aplicación de los criterios de inclusión y exclusión, fueron seleccionados 9 artículos indexados en las bases de datos LILACS y BDENF, en el período de 2006 a 2017. Los resultados evidenciaron que la educación en salud proporciona innumerables beneficios, sin embargo, no es una tarea fácil, ya que no se limita sólo a la transmisión de informaciones a las usuarias, sino de un proceso educativo dialógico en la búsqueda de autonomía. Consideraciones finales: Para ello, se hace importante la adopción de estrategias y prácticas metodológicas activas, además de la concientización de los profesionales sobre la importancia de la educación en salud en el proceso de humanización de la asistencia.

Palabras clave: Educación en salud, Asistencia prenatal y Enfermería.

\section{INTRODUÇÃO}

O acesso de gestantes ao pré-natal é um importante fator no prognóstico ao nascimento, pois durante esse acompanhamento é assegurado o desenvolvimento de uma gestação segura, permitindo um parto sem intercorrências à saúde materna e a gestação de um recém-nascido saudável (BRASIL, 2012).

Um instrumento relevante que auxilia os profissionais no ciclo gravídico é a educação em saúde, pois tem por objetivo conscientizar a usuária sobre as necessidades de saúde e evitar possíveis complicações. Nesse sentido, as práticas de vida saudáveis são estimuladas, incluindo melhorias no autocuidado. Logo, a educação em saúde torna-se uma possibilidade para uma rotina saudável na vida da gestante (COSTA FDS, et al., 2008; OLIVEIRA RL e SANTOS MEA, 2011; BRASIL, 2012).

Desde modo, faz-se necessário a criação de espaços de educação em saúde para o pré-natal objetivando a interação entre as gestantes e profissionais, e em muitos casos, intervenções junto à família e à comunidade geram impactos positivos (BRASIL,2012).

Nestes espaços, as mulheres podem interagir, ouvirem e falarem de suas vivências e experiências, consolidando informações importantes sobre os assuntos referentes à gestação, parto e puerpério. Também pode-se desenvolver grupos específicos para gestantes, em salas de espera, atividades em comunidades e escolas ou em outros espaços de trocas de ideias e compartilhamento de saberes dos mais variados possíveis na busca de soluções das mais diversas problemáticas (COSTA FDS et al,2008).

Na prática, é notável importância que o pré-natal, de qualidade, tem na evolução de uma gestação e no desfecho favorável da mesma. Ainda há muitos profissionais que atuam somente nas necessidades fisiopatológicas e poucos consideram os processos educativos em saúde como instrumento de promoção à saúde às gestantes e seus acompanhantes (DUARTE SJH e ANDRADE SMOD,2008).

Cabe aos profissionais, dentre eles, o enfermeiro, compreender esta realidade e estarem sensibilizados para uma assistência humanizada e integral no transcorrer do pré-natal, reduzindo os riscos materno-fetal no período gestacional conforme Duarte SJH e Andrade SMOD (2008), desenvolvendo a promoção da saúde e a prevenção de doenças (OLIVEIRA RL e SANTOS MEA,2011).

Porém, o que se observa nos serviços de saúde, em sua grande maioria, é que a assistência oferecida às gestantes não responde aos anseios em sua totalidade, já que nem sempre os profissionais são capacitados para o desenvolvimento de educação em saúde. Sendo assim, faz-se necessário buscar, na literatura, as evidências científicas que corroborem com a importância do desenvolvimento de educação em saúde na assistência pré-natal para subsidiar a Prática Fundamentada em Evidência (PBE). Para tanto, apresenta-se o seguinte questionamento: qual a importância da educação em saúde na assistência pré-natal, como a equipe multiprofissional utiliza esta ferramenta e quais dificuldades emergem das práticas educativas em saúde?

Baseado nestas perspectivas, o objetivo do estudo é: apresentar a importância da educação em saúde, como a equipe multiprofissional a utiliza e as dificuldades que emergem dessa prática na assistência prénatal, segundo as publicações científicas.

REAS/EJCH | Vol. Sup. 23 | e397 | DOI: https://doi.org/10.25248/reas.e397.2019 Página 2 de 8 


\section{MÉTODOS}

A metodologia abordada no presente estudo é uma Revisão Narrativa da Literatura (RNL). Realizou-se, inicialmente, um levantamento de produções científicas, por meio de fontes documentais em bases de dados virtuais, Manuais do Ministério da Saúde, portarias, além de consulta a bibliografias impressas.

O levantamento literário forneceu um acervo de documentos tais como cadernos, portarias e políticas do Ministério da Saúde, publicados no período de 2005 a 2012 e dissertações. Os artigos selecionados foram publicados no período de 2006 a 2017, orientando a realização do embasamento teórico.

A busca dos artigos ocorreu por meio da Biblioteca Virtual em Saúde (BVS), no período de Novembro de 2017 a Janeiro de 2018, nas referidas bases de dados: Medical Literature Analysis and Retrieval System Online (MEDLINE), Latino-americano em Ciências da Saúde (LILACS), Scientific Eletronic Library Online (SCIELO), Base de Dados da Enfermagem (BDENF). Foram utilizados Descritores em Ciências da Saúde (DeCS), a saber: Educação em saúde, Assistência Pré-Natal e Enfermagem.

Para a pesquisa em base de dados aplicou-se os seguintes critérios de inclusão: pesquisas primárias; produções disponíveis online; formato de artigos científicos completos; produções relacionadas à temática em questão; publicados em português; compreendendo o período de 2006 a 2017, existentes na Literatura MEDLINE, Latino-americano em Ciências da Saúde (LILACS), Scientific Eletronic Library Online (SCIELO) e Base de Dados da Enfermagem (BDENF). Foram excluídos do presente estudo, as revisões de literatura e as publicações que não atenderam aos critérios acima citados.

\section{RESULTADOS}

A partir da busca em bases de dados foram identificadas 748 publicações, sendo 533 na MEDLINE, 90 na LILACS, 94 na BDENF e 0 na SCIELO. Posteriormente, foram aplicados os critérios de inclusão, restandoIhes 92 artigos. Posteriormente foi realizada a leitura por título e resumo, restando 18 artigos, e em seguida foi realizada a leitura na integra dos artigos, chegando a uma amostra final de 09 artigos que foram incluídos na revisão literária somados aos demais documentos.

Os anos com maior número de publicações foram 2011, 2014 e 2016, todos com duas publicações, e os demais (2006, 2010 e 2013), apenas com uma publicação cada. No que se refere ao perfil metodológico das publicações, todos os 09 artigos eram de caráter descritivo, 08 artigos de abordagem qualitativa e apenas 01 de abordagem quanti-qualitativa, como demonstrado a seguir, no Quadro 1.

Em relação à categoria profissional, a enfermagem é a predominante, sendo referenciado em 08 dos 09 artigos utilizados. Outras categorias abordadas nos estudos foram: Nutrição, Fisioterapia e Medicina (01 artigo cada).

De acordo com os artigos selecionados, observou-se os benefícios da educação em saúde no pré-natal foram abordados em 07 dos 09 artigos utilizados nesta análise. Sendo os principais: o aprendizado; a minimização de medos e dúvidas; a troca de experiências; o vínculo entre profissional de saúde e usuárias e autonomia da mulher como ser ativo no autocuidado.

Sobre as estratégias utilizadas na prática educativa foi constatado que o grupo de gestantes foi o mais abordado, sendo incluído em 07 artigos, seguido dos jogos educativos e utilização de brinquedos com 01 artigo cada. No quadro 2, a seguir, foram agrupados os artigos selecionados de acordo com o ano, população de estudo, objetivo e resultados obtidos.

Baseado nestas fontes de pesquisa, a revisão de literatura na busca de mostrar os embasamentos de evidências científicas acerca da importância da educação em saúde na assistência pré-natal, as estratégias utilizadas pela equipe multiprofissional e as dificuldades que emergem das práticas educativas em saúde. 
Os principais resultados encontrados nos acervos selecionados foram discutidos nas seguintes categorias, a saber: A importância da educação em saúde na assistência pré-natal e o papel do enfermeiro; Estratégias e metodologias utilizadas nas práticas educativas em saúde no pré-natal e dificuldades/fragilidades na educação em saúde no pré-natal.

Quadro 1 - Resultado da pesquisa nas bases de dados LILACS e BDENF.

\begin{tabular}{|c|c|c|c|c|}
\hline BASES & PERIÓDICOS & ANO & TÍTULO & AUTORES \\
\hline LILACS & $\begin{array}{l}\text { Online Brazilian } \\
\text { Journal of } \\
\text { Nursing }\end{array}$ & 2006 & $\begin{array}{l}\text { Curso para gestantes: ação } \\
\text { educativa na perspectiva da co- } \\
\text { responsabilidade. }\end{array}$ & $\begin{array}{l}\text { Rolim MO.; } \\
\text { Moreira TMM.; } \\
\text { Viana GRO, } 2006 .\end{array}$ \\
\hline LILACS & $\begin{array}{l}\text { Rev. Eletr. Enf. } \\
\text { [Internet] }\end{array}$ & 2010 & $\begin{array}{l}\text { Socialização de conhecimentos } \\
\text { e experiências sobre o processo } \\
\text { de nascimento e tecnologias do } \\
\text { cuidado. }\end{array}$ & Darós DZ. et al., 2010. \\
\hline LILACS & $\begin{array}{l}\text { Rev. Eletr. Enf. } \\
\text { [Internet] }\end{array}$ & 2011 & $\begin{array}{l}\text { Ações educativas durante a } \\
\text { assistência pré-natal: percepção } \\
\text { de gestantes atendidas na rede } \\
\text { básica de Maringá-PR. }\end{array}$ & $\begin{array}{l}\text { Souza VB de; } \\
\text { Roecker S; } \\
\text { Marcon SS, } 2011 .\end{array}$ \\
\hline BDENF & Rev. Rene & 2011 & $\begin{array}{l}\text { Contribuições do pré-natal para o } \\
\text { parto vaginal: percepção de } \\
\text { puérperas }\end{array}$ & Costa AP. et al., 2011. \\
\hline LILACS & $\begin{array}{l}\text { Rev. Enferm. } \\
\text { UERJ }\end{array}$ & 2013 & $\begin{array}{l}\text { Aplicação de tecnologia leve no } \\
\text { pré-natal: um enfoque na } \\
\text { percepção das gestantes }\end{array}$ & Alves ACP et al., 2013 \\
\hline LILACS & $\begin{array}{l}\text { Rev Bras. } \\
\text { Enferm. }\end{array}$ & 2014 & $\begin{array}{l}\text { Educação em saúde no ciclo } \\
\text { gravídico-puerperal: sentidos } \\
\text { atribuídos por puérperas. }\end{array}$ & $\begin{array}{l}\text { Guerreiro EM et al., } \\
2014 .\end{array}$ \\
\hline BDENF & $\begin{array}{l}\text { Rev Enferm } \\
\text { UFSM }\end{array}$ & 2014 & $\begin{array}{l}\text { Motivos e repercussões da } \\
\text { participação de gestantes em } \\
\text { grupo operativo no pré-natal. }\end{array}$ & Maron LC et al., 2014. \\
\hline LILACS & Rev Rene. & 2016 & $\begin{array}{l}\text { Enfermagem obstétrica e } \\
\text { educação em saúde: } \\
\text { contribuições para vivência do } \\
\text { processo de parturição. }\end{array}$ & $\begin{array}{l}\text { Quadros JS; Reis T } \\
\text { LR. dos; Colomé JS, } \\
\text { 2016. }\end{array}$ \\
\hline BDENF & $\begin{array}{l}\text { Jornal Of Nursing } \\
\text { And Health. }\end{array}$ & 2016 & $\begin{array}{l}\text { Percepção das gestantes sobre } \\
\text { as consultas de pré-natal } \\
\text { realizadas pelo enfermeiro na } \\
\text { atenção básica. }\end{array}$ & $\begin{array}{l}\text { Campos ML de. et al., } \\
2016 \text {. }\end{array}$ \\
\hline
\end{tabular}

Fonte: Elaborado pela autora, de acordo com os artigos selecionados nos anos de 2006 a 2017. 
Quadro 2. Principais produções científicas elencadas e procedimentos metodológicos.

\begin{tabular}{|c|c|c|c|c|}
\hline Autor & Ano & População & Objetivo & Resultados \\
\hline $\begin{array}{l}\text { ROLIM MO et } \\
\text { al. }\end{array}$ & 2006 & $\begin{array}{l}09 \text { grávidas que obedeceram } \\
\text { aos critérios de inclusão. }\end{array}$ & $\begin{array}{l}\text { Avaliar a eficácia de um curso para gestantes, } \\
\text { desenvolvido na perspectiva da co- } \\
\text { responsabilidade, em uma Unidade Básica de } \\
\text { Saúde da Família em São Gonçalo do } \\
\text { Amarante/Ceará. }\end{array}$ & $\begin{array}{l}\text { Todas as gestantes referiram ter mudado algo em } \\
\text { sua vida após participarem do curso de gestante. }\end{array}$ \\
\hline DARÓS et al. & 2010 & $\begin{array}{l}09 \text { gestantes, parturientes, } \\
\text { puérperas, usuárias da unidade } \\
\text { básica de saúde (ULS) do bairro } \\
\text { dos Ingleses, no município de } \\
\text { Florianópolis, que participaram } \\
\text { de um processo educativo } \\
\text { durante o terceiro trimestre de } \\
\text { gestação em seu domicílio. }\end{array}$ & $\begin{array}{l}\text { Conhecer a importância do processo } \\
\text { educativo e as contribuições ao socializar } \\
\text { conhecimentos sobre o processo de } \\
\text { nascimento e ao praticar tecnologias de } \\
\text { cuidado na gestação. }\end{array}$ & $\begin{array}{l}\text { Facilitou a escolha de determinado tipo de parto e } \\
\text { a decisão sobre o uso das tecnologias que } \\
\text { julgassem mais confortáveis. }\end{array}$ \\
\hline ALVES et al. & 2013 & $\begin{array}{l}17 \text { gestantes em duas unidades } \\
\text { básicas de saúde do município } \\
\text { de Brejo Santo-CE. }\end{array}$ & $\begin{array}{l}\text { Identificar as percepções das gestantes sobre } \\
\text { o uso de uma tecnologia educativa para ser } \\
\text { utilizada no pré-natal. }\end{array}$ & $\begin{array}{l}\text { A estratégia permitiu uma maior espontaneidade } \\
\text { para expor experiências, retirar dúvidas e oferecer } \\
\text { novas informações }\end{array}$ \\
\hline $\begin{array}{l}\text { GUERREIRO } \\
\text { et al. }\end{array}$ & 2014 & $\begin{array}{l}31 \text { usuárias dos Centros de } \\
\text { Saúde da Família de Fortaleza- } \\
\text { CE, maiores de } 18 \text { anos, no } \\
\text { puerpério, no mínimo seis } \\
\text { consultas de pré-natal e uma } \\
\text { consulta puerperal. }\end{array}$ & $\begin{array}{l}\text { Apreender os conteúdos das representações } \\
\text { sociais de puérperas sobre a educação em } \\
\text { saúde no ciclo gravídico-puerperal na atenção } \\
\text { básica de saúde. }\end{array}$ & $\begin{array}{l}\text { A educação em saúde objetiva-se nas palestras, } \\
\text { campanhas educativas, e ainda nas atividades } \\
\text { voltadas para a educação do filho, em espaços } \\
\text { sócio familiares como a casa ou a escola. }\end{array}$ \\
\hline MARON et al. & 2014 & $\begin{array}{l}\text { Seis gestantes, destas, três } \\
\text { primigestas e três } \\
\text { secundigestas e dois } \\
\text { companheiros das mesmas, } \\
\text { totalizando oito sujeitos. }\end{array}$ & $\begin{array}{l}\text { Conhecer as motivações de mulheres-mães e } \\
\text { familiares para a participação em grupo } \\
\text { operativo de gestantes e identificar a } \\
\text { repercussão de sua participação nessa } \\
\text { atividade. }\end{array}$ & $\begin{array}{l}\text { As mulheres-mães se sentem inexperientes para o } \\
\text { cuidado com o bebê, dada à singularidade do } \\
\text { processo de nascimento. }\end{array}$ \\
\hline $\begin{array}{l}\text { CAMPOS et } \\
\text { al. }\end{array}$ & 2016 & $\begin{array}{l}\text { Seis mulheres, sendo cinco } \\
\text { gestantes e uma puérpera. }\end{array}$ & $\begin{array}{l}\text { Conhecer a percepção das gestantes sobre as } \\
\text { consultas de pré-natal realizadas pelo } \\
\text { enfermeiro na atenção básica. }\end{array}$ & $\begin{array}{l}\text { No que se refere às ações educativas, foi possível } \\
\text { perceber que foram voltadas para problemas ou } \\
\text { formas de evitá-los, e somente quando solicitadas, } \\
\text { existindo uma falta de orientações quanto ao parto, } \\
\text { aos cuidados com o recém-nascido, e à } \\
\text { amamentação. }\end{array}$ \\
\hline
\end{tabular}

Fonte: Elaborado pela autora, de acordo com os artigos selecionado nos anos de 2006 a 2017. 


\section{DISCUSSÃO}

\section{A importância da educação em saúde na assistência à gestante e o papel da equipe multidisciplinar}

A integralidade da assistência engloba a articulação contínua da promoção da saúde com prevenção e tratamento de agravos por ações prestadas, indididualmente ou coletivamente Souza MCD et al. (2012), envolve ato de cuidar das pessoas por meio de ações coesas e interligadas e o incentivo às práticas de promoção de saúde e prevenção, sem que haja prejuízos das práticas assistenciais (SOUZA,VBD et al.2011).

Para Rolim, et al. (2006) as ações educativas são fundamentais por ampliar conhecimentos, aprofundar discussões, de forma que as gestantes e puérperas possam superar suas dificuldades, obtendo maior autonomia e podendo viver de maneira mais harmônica.

Além do aprendizado proporcionado pela prática educativa, há também, troca de experiências entre os membros envolvidos no grupo educativo, a formação de laços de amizade entre gestantes participantes e o estreitamento do vínculo entre usuária e professional( MARON LC, et al.2014).Vínculo que gera uma atmosfera de confiança e respeito conforme Darós DZ, et al.(2010) minimizando as inseguranças e anseios que possam surgir durante o período gravídico-puerperal (GUERREIRO M, et al.2014).

Quando a gestante é incluída neste processo viabiliza-se oportunidade do diálogo e permite à mesma ser multiplicadora de saúde no seu coletivo, propicia ainda socialização com seus pares e promoção da conscientização e participação nas decisões, visando à transformação das suas limitações (SOUZA VBD, et al.2011).

É importante enfatizar as práticas educativas como responsabilidade de uma equipe multiprofissional, contribuindo com a qualidade do fazer no cotidiano do profissional e com a interação de conhecimentos entre os membros da equipe e entre profissionais e usuários, seja no individual ou no coletivo, havendo o planejamento conjunto de ações que visem transformar uma determinada realidade (PAULA RAD,2017).

Entre os profissionais de saúde, o enfermeiro tem um papel fundamental no que se refere à educação em saúde. As orientações prestadas pelo enfermeiro podem reduzir os medos, ansiedade e facilitar o processo de gestação e nascimento, fazendo com que a gestante assuma o papel de protagonista, participante ativamente do processo ensino-aprendizagem (CAMPOS ML, et al.2016).

Mulheres que tiveram acompanhamento de enfermeiros e/ou residentes em enfermagem obstétrica durante o ciclo gravídico-puerperal sentiram-se mais confiantes e preparadas para a experiência do parto e cuidados com o recém-nascido, por conta das orientações que foram repassadas. Além que, as ações educativas desenvolvidas por enfermeiros e/ou residentes em enfermagem obstétrica viabilizaram recepção e acolhimento seguro para o futuro recém-nascido (QUADRIS SD, et al.2016).

Espera-se que as equipes de saúde possam trabalhar a educação em saúde de forma sistemática em todos os níveis de atenção, contribuindo no sentido de resgatar e consolidar a cidadania como direito de todos, proporcionando um cuidado voltado para a integralidade do ser humano, oferecendo subsídios para o desempenho da autonomia da gestante e sua família.

\section{As estratégias e metodologias utilizadas nas práticas educativas}

Existem inúmeras formas de realizar um trabalho educativo, destacando-se as discussões em grupo, conhecidos popularmente como grupo de gestantes. Esta metodologia educativa pode ser bastante explorada, pois objetiva a promoção da saúde e consequentemente a prevenção de doenças, buscando a participação dos sujeitos na concfretização do autocuidado (GUERREIRO M, et al. 2014).

As gestantes que participaram de grupos estavam mais seguras sobre o processo gestacional e aos cuidados pós-parto consigo e com o bebê. Para as gestantes as ações educativas em grupo foram 
importantes no sentido de esclarecer as dúvidas e reduzir medos e a ansiedade na gestação de acordo com Maron LC, et al. (2014).

Além dos grupos de gestantes, outra metodologia que pode ser lançada para a prática educativa é a utilização de jogos. O jogo educativo surge como uma estratégia inovadora para ser utilizada durante o prénatal, proporcionando uma participação ativa das gestantes e permitindo uma fixação maior do assunto abordado devido à interação dinâmica entre facilitador e gestante (ALVES CP, et al.2013).

A utilização de brinquedos para trabalhar os cuidados com bebe demonstrou-se bastante favorável, pois as gestantes visualizavam a forma como deveriam proceder durante determinada ação, permitindo desenvolver a atividade prática, bem como proporcionar um ambiente adequado para conhecer melhor a forma correta de cuidar do recém-nascido e retirar as dúvidas (ALVES CP, et al.2013).

As palestras possuem seu valor enquanto metodologia nas ações educativas, porém elas estabelecem limites para o aprendizado por não proporcionar a interações entre as usuárias e profissionais, tornando-se algo mecânico e dependente da didática do palestrante (GUERREIRO M, et al.2014).

\section{As dificuldades/fragilidades na educação em saúde}

A educação em saúde é uma excelente ferramenta para promoção da saúde, porém, ainda se observa uma carência na sua utilização. Logo, torna-se um desafio introduzir educação em saúde na prática assistencial. Segundo os autores, alguns profissionais estão acostumados a trabalhar de maneira assistencialista, já que, segundo os profissionais, a educação em saúde necessita de persistência e prática diária para ser observado algum tipo de resultado efetivo (QUADROS SD, et al.2016).

Segundo um estudo, em cinco UBS, não houve uma atuação constante e contínua dos enfermeiros em ações educativas. Foi percebido que os profissionais continuam sendo pouco acolhedores e consequentemente não há a formação de vínculos com as gestantes acompanhadas por estes profissionais (SOUZA VBS, et al.2011).

Nos achados houve insatisfação das gestantes devido à falta de cordialidade nos serviços de saúde, atendimentos rápidos, dificultando o diálogo com o profissional de saúde e impedindo que as mulheres demonstrassem suas queixas, dúvidas e medos. Os profissionais eram tidos como incompreensivos, indiferentes e grosseiros, comprometendo a qualidade da assistência e promovendo um sofrimento psíquico às gestantes (COSTA A, et al.2011).

A participação social é algo que se faz necessário na análise e na formulação de ações que visem à sua promoção. Assim, a abordagem de promoção da saúde aponta para o desenvolvimento de políticas públicas e para a produção e disseminação de conhecimentos e práticas de saúde de forma compartilhada e participativa (BRASIL,2006).

Existe a necessidade de capacitação permanente dos profissionais, no sentindo de integrar às ações de promoção e prevenção, levando em conta as peculiaridades e necessidades específicas desta clientela para proporcionar uma melhor qualidade de vida durante e após a gestação (SOUZA VBD, et al.2011)

Em relatos de puérperas, é nítido a desinformação sobre o processo de parturição, apresentando dificuldade em reconhecer os sinais de início do trabalho de parto e alterações clínicas que necessitam de atendimento de saúde. Além que, muitas mulheres ainda desconhecem sobre o direito ao acompanhante durante o trabalho de parto e nascimento, mesmo sendo uma prática respaldada por lei desde 2005 (QUADROS SD, et al.2016).

O pré-natal deve ser organizado de forma atender às necessidades das gestantes e para tal faz-se necessário dispor de profissionais com conhecimentos técnico-científicos, que dão enfoque à humanização durante $o$ atendimento, onde a prática deverá estar baseada na integralidade das ações. As orientações não 
devem ser voltadas apenas para prevenção ou resolução de problemas e nem serem fornecidas apenas quando solicitadas pelas gestantes (CAMPOS ML, et al 2016; SIMÕES ALDA, et al.2007).

Além disso, a "alta do pré-natal" antes do período preconizado é um fator negativo para a assistência da gestante pois, em sua maioria, existe uma falha no acompanhamento ao final da gestação, aumentando o risco de complicações obstétricas (BRASIL, 2005).

\section{CONSIDERAÇÕES FINAIS}

A presente revisão de literatura evidenciou que a educação em saúde é imprescindível na assistência prénatal. Além disso, inúmeras são as estratégias que poderão ser adotadas na produção de um cuidado integral à gestante, como os jogos educativos, grupos de gestantes e o uso de brinquedos no compartilhamento de conhecimentos e experiências acerca do trabalho de parto, cuidados com o recém-nascido e aleitamento materno. Evidenciou ainda os principais desafios a serem superados junto aos profissionais de saúde, principalmente pelo enfermeiro, como a não priorização da educação em saúde durante a prestação da assistência; a falta de condições necessárias e capacitação permanente dos profissionais de saúde e a insatisfação das gestantes com estes profissionais. Logo, deve-se ocorrer uma mudança no enfoque puramente assistencialista, promovendo saúde e prevenção de agravos por meio de práticas educativas que proporcionam inúmeros benefícios de aprendizado, superação de medos e anseios, troca de experiências, a formação de vínculo entre usuárias e profissional de saúde e obtenção de autonomia da mulher, garantindo à gestante uma assistência de qualidade, humanizada e integral.

\section{REFERÊNCIAS}

1. Alves CP, Figueiredo dFER, Sousa PLd, Oliveira Jd, Oliveira Rd, Sousa WMd. Aplicação de tecnologia leve no pré-natal: um enfoque na percepção das gestantes. Rev. enferm. UERJ. 2013 dez; 21(1).

2. Campos ML, Veleda AA, Coelho DF, Telo SV. Percepção das gestantes sobre as consultas de pré-natal realizadas pelo enfermeiro na atenção básica. Jounal of Nursing and Health. 2016; 6(3).

3. Costa A, Bustorff ACV, Cunha ARRd, Soares MCdS, Araújo VS. Contribuições do pré-natal para o parto vaginal: Percepção de puérperas. Rev Rene. 2011 jul/set; 12(2).

4. Costa FdS, Silva JLLd, Diniz MIG. A IMPORTÂNCIA DA INTERFACE EDUCAÇÃOISAÚdE NO AMBIENTE ESCOLAR. Informe-se em promoção da saúde. 2008: p. 30-33.

5. Darós DZ, Hess PT, Sulsbach P, Zampieri MdFM, Daniel HS. Socialização de conhecimentos e experiências sobre o processo de nascimento e tecnologias do cuidado. Rev. Eletr. Enf. [Internet]. $2010 \mathrm{Fev} / \mathrm{Jun} ;$ 12(2).

6. Duarte SJH, Andrade SMOd. O Significado do Pré-Natal para Mulheres Grávidas: uma experiência no município de Campo Grande, Brasil. Saúde Soc. 2008: p. 132-139.

7. Guerreiro M, Rodrigues P, Queiroz BA, Ferreira dA. Educação em saúde no ciclo gravídico-puerperal: sentidos atribuídos por puérperas. Rev Bras Enferm. 2014 jan/fev; 67(1).

8. Maron LC, Cabral FB, Sand ICPVd, Hildebrandt LM. Motivos e repercussões da participação de gestantes em grupo operativo no pré-natal. Rev enferm UFSM. 2014 Jul/Set; 4(3).

9. Ministério da Saúde. Departamento de Atenção Básica. [Atenção ao pré-natal de baixo risco].; 2012 [cited 2017 Dezembro 13. Available from: http://bvsms.saude.gov.br/bvs/publicacoes/cadernos atencao basica 32 prenatal.pdf.

10. Ministério da Sáude. Departamento de Ações Programáticas Estratégicas. [Pré-natal e puerpério: atenção humanizada e

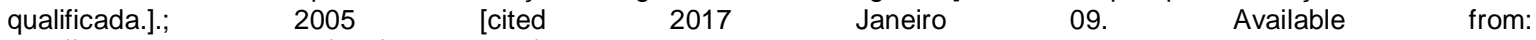
http://bvsms.saude.gov.br/bvs/publicacoes/manual pre natal puerperio 3ed.pdf.

11. Ministério da Saúde. Secretaria de Vigilância à Saúde. Política Nacional de Promoção da Saúde: revisão da Portaria MS/GM ํo 687, de 30 de março de 2006.

12. Oliveira RL, Santos MEA. Educação em saúde na estratégia saúde da família: Conhecimentos e práticas do enfermeiro. Revista Enfermagem Integrada. 2011 Nov/Dez; 4(2).

13. Paula RAd. [Relação multiprofissional do trabalho em equipe na atenção básica de saúde]. [cited 2017 Novembro 15. Available from: https://www.nescon.medicina.ufmg.br/biblioteca/imagem/0296.pdf.

14. Quadros Sd, Reis TLdRd, Colomé S. QUADROS, J. S. de; REIS, T. L. daEnfermagem obstétrica e educação em saúde: contribuições para vivência do processo de parturição. Rev Rene. 2016 jul/ago; 17(4).

15. Rolim MO, Moreira TMM, Viana GRO. Curso para gestantes: ação educativa na perspectiva da co-responsabilidade. Online Brazilian Journal of Nursing. 2006 Out/Nov; 5(3).

16. Souza MCd, Araújo TMd, Júnior WMR, Souza JN, Vilela ABA, Franco TB. Integralidade na atenção à saúde: um olhar da Equipe de Saúde da Família sobre a fisioterapia. O Mundo da Saúde. 2012; 36(3).

17. Souza VBd, Roecker S, Marcon SS. Ações educativas durante a assistência pré-natal: percepção de gestantes atendidas na rede básica de Maringá-PR. ev. Eletr. Enf. [Internet]. 2011 abr/jun; 12(2).

18. Simões ALdA, Bittar DB, Mattos ÉF, Sakai LA. A humanização do atendimento no contexto atual de saúde: Uma reflexão. REME - Rev. Min. Enf. 2007 Jan/Mar; 11(1). 